\title{
MicroRNA-449a Inhibits Proliferation and Induces Apoptosis by Directly Repressing E2F3 in Gastric Cancer
}

\author{
Xiaoping Lia Hong Lib Rui Zhang ${ }^{\mathrm{c}}$ Jing Liü Jun Liuc \\ aDepartment of Clinical Laboratory, Wujiang first people's hospital Affiliated Nantong University, \\ Suzhou, ${ }^{b}$ Department of Clinical Laboratory, The Second Affiliated Hospital of Shaanxi University of \\ Chinese Medicine, Shaanxi University of Chinese Medicine, Xianyang, 'Department of Oncology, The \\ Taixing People's Hospital, Taixing, dDepartment of Pathology, The Taixing People's Hospital, Taixing, \\ People's Republic of China
}

\section{Key Words}

Gastric cancer • MiR-449a • E2F3 • Proliferation • Apoptosis

\begin{abstract}
Background: MicroRNA-449a is a tumor suppressor that is down-regulated in multiple tumors types. However, the role of miR-449a in gastric cancer (GC) remains largely unknown. Methods: MiR-449a expression was up-regulated using miR-449a mimics, and the role of miR449a in GC was assessed using cell viability and apoptosis assays. miR-449a target genes were confirmed using luciferase activity, RT-PCR and western blot assays. Results: miR-449a was downregulated in gastric cancer cell lines and gastric cancer tissues. Restoration of miR-449a expression inhibited gastric cancer cell proliferation and colony formation. Significant G0/G1 arrest was observed in gastric cancer cells transfected with miR-449a mimics. Furthermore, combination therapy with miR-449a with cisplatin displayed greater anti-tumor effects than treatment with cisplatin alone. We also identified E2F3 (E2F transcription factor 3), an important transcription factor involved in the proliferation and metastasis of tumor cells, as a direct target gene of miR-449a. Furthermore, silencing E2F3 elicits similar a repressive effect as overexpression of miR-449a in gastric cancer cells, and E2F3 overexpression rescued the repressing effects of miR-449a mimics. Conclusions: This study indicates that the miR-449a/ E2F3 axis plays an important role in proliferation and apoptosis in gastric cancer. Therefore, miR-449a represents a novel target for gastric cancer therapy.
\end{abstract}




\section{Introduction}

Gastric cancer (GC) is one of the most common types of malignancies around the world. Over the past 20 years, the incidence and mortality rate of GC have decreased worldwide, but it still ranks as the second leading cause of cancer death and the most common gastrointestinal malignancy in East Asia [1]. Despite recent advances in gastrectomy, chemotherapy, and radiotherapy, more than half of all advanced stage GC patients die of recurrence, even after undergoing curative gastrectomy [2]. Therefore, elucidation of the regulatory mechanisms modulating GC tumorigenesis and the molecular alterations underlying this disease is imperative for developing novel targeted therapies for gastric cancer [3].

MicroRNAs (miRNAs) are a family of small, non-coding RNAs that negatively regulate the expression of target genes by altering mRNA translation or stability [4]. miRNAs play important roles in a wide variety of physiological or pathological processes, including tumorigenesis. An increasing body of evidence indicates that microRNA expression is dysregulated in human cancers, implying an essential role for miRNAs during tumor progression [5]. MiRNAs function as regulatory molecules, acting as oncogenes or tumor suppressor genes in tumorigenesis. Furthermore, abnormal miRNA expression is associated with the development and progression of GC [6]. However, the exact molecular mechanisms by which miRNAs influence GC remain unclear. The role of miRNAs in GC tumorigenesis warrants further investigation.

MicroRNA-449a (miR-449a) is an important miRNA that has been identified in previous cancer studies. MiR-449a is acts as tumor suppressor, and it is downregulated in various types of cancer, including gastric cancer [7]. However, little is known about the role of miR449a in GC tumorigenesis and progression. The aim of the present study is to explore the role of miR-449a in GC cell proliferation and apoptosis.

\section{Materials and Methods}

Tissue sample collection and cell culture

Twelve primary gastric cancer tissue samples and non-tumor gastric tissues were collected from Wujiang first people's hospital. Written informed consent was obtained from each participant. This study was approved by the institutional review board of Wujiang first people's hospital. Tissue samples were quickly frozen in liquid nitrogen immediately after surgical removal and stored at $-80^{\circ} \mathrm{t}$. Normal human gastric epithelial cells (NGEC) and the gastric cancer cell line, SGC-7901, were obtained from the Institute of Biochemistry and Cell Biology, Chinese Academy of Sciences (Shanghai, China). The cells were maintained in DMEM medium, supplemented with $10 \%$ fetal bovine serum (FBS; Hyclone, USA), $100 \mathrm{U} / \mathrm{mL}$ penicillin, and $100 \mu \mathrm{g} / \mathrm{mL}$ streptomycin. The cells were incubated in an atmosphere of $5 \% \mathrm{CO}_{2}$ at $37^{\circ} \mathrm{C}$. Primary normal human gastric epithelial cells (NGEC) were established as described previously [8].

\section{Synthesis and transfection of miRNA/siRNA and plasmid DNA}

miR-449a mimics, negative controls (NC), and siRNA targeting human E2F3 mRNA (GenBank no. NM. 001949) were designed and synthesized by Shanghai GenePharma Company (Shanghai, China). For transfection, $4 \times 10^{3}$ cells per well were seeded into 96 -well plates in antibiotic-free growth medium. Once the cells reached $80-90 \%$ confluence, they were transfected with human miR-449a mimics or negative control (NC). For E2F3 or scrambled siRNA, cells were transfected at 30-40\% confluence using Lipofectamine 2000 (Invitrogen, USA), according to the manufacturer's protocol. The pCDNA3.1-E2F3 plasmid, which encodes the entire E2F3 coding sequence but lacks the normal 3'-untranslated sequence, was purchased from Houzai Biotech Company (Nanjing, China). This plasmid was transfected as described previously [9]. Experiments were performed $72 \mathrm{~h}$ after transfection.

RNA extraction and quantitative reverse transcription-PCR ( $q R T-P C R$ )

To quantitate the expression of miR-449a, total RNA, including miRNA, was extracted from the cell lines and tissue samples using TRIzol reagent (Life Technologies, Inc., Carlsbad, CA, USA). RNA was synthesized into cDNA using M-MuLV Reverse Transcriptase (Fisher Scientific, Pittsburgh PA, USA). Real-time PCR was 


\section{Cellular Physiology Cell Physiol Biochem 2015;35:2033-2042

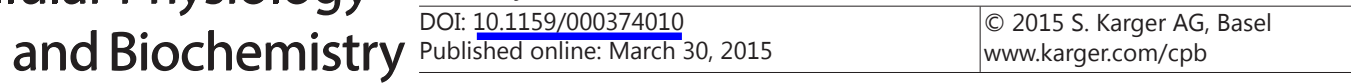

Li et al.: MicroRNA-449a Inhibits Proliferation and Induces Apoptosis in Gastric Cancer

conducted using SYBR Green mix (Takara, Dalian, China) and a CFX96 real-time PCR detection system (BioRad, Hercules, CA, USA) under the following conditions: $94{ }^{\circ} \mathrm{C}$ for $3 \mathrm{~min}$, followed by 35 cycles of $94{ }^{\circ} \mathrm{C}$ for 30 $\mathrm{s}$ and $60^{\circ} \mathrm{C}$ for $30 \mathrm{~s}$. The expression of miR-449a was normalized to U6 RNA and calculated using the $2^{-\Delta \Delta \mathrm{Ct}}$ method. E2F3 mRNA levels were detected as described previously [10].

\section{Cell proliferation assay}

Cell proliferation was determined using the Cell Counting Kit-8 assay kit (Takara, Dalian, China), according to the manufacturer's instructions. Cells were seeded at a density of $3 \times 10^{4}$ per well in 96 -well plates, and each well was transfected with miR-449a mimic (50 nM), inhibitor (100 nM) or the proper negative control. Seventy-two hours after transfection, the absorbance was read at $450 \mathrm{~nm}$ using an iMark Microplate Absorbance Reader (Bio-Rad, Hercules, CA, USA).

\section{Cell cycle assay}

The cell cycle assay was performed as previously described [11]. Seventy-two hours after transfection, cells were harvested and washed twice at $4{ }^{\circ} \mathrm{C}$ with PBS containing $0.5 \%$ BSA. Cells were collected, resuspended in $300 \mu \mathrm{l}$ PBS, and fixed by adding $700 \mu \mathrm{l}$ of $100 \%$ ethanol at $4{ }^{\circ} \mathrm{C}$ for $24 \mathrm{~h}$. Cells were washed twice in ice cold PBS to remove ethanol and then resuspended in a propidium iodide (PI) solution containing $100 \mu \mathrm{g} / \mathrm{ml}$ PI and $50 \mu \mathrm{g} / \mathrm{ml}$ RNase (Sigma, USA) at $37^{\circ} \mathrm{C}$ for $30 \mathrm{~min}$ in the dark. Cell number in each phase of the cell cycle was determined using Calibur Flow Cytometers and analyzed with CELL Quest software.

\section{Analysis of clonogenicity in vitro}

Aliquots of viable SGC-7901 cells (1,000 per well) transfected with miR-449a mimic or NC were placed in six-well plates $24 \mathrm{~h}$ after transfection and maintained in DMEM medium supplied with $10 \%$ fetal bovine serum for 2 weeks. The number of colonies containing more than 50 cells were stained with crystal violet and counted. Triplicate wells were measured for each group.

\section{Apoptosis analysis by annexin-V FITC/PI double staining}

The cells were treated with $5 \mu \mathrm{M}$ cisplatin. To identify apoptotic cells, annexin V and PI staining was performed using an Annexin V-FITC Apoptosis Detection kit (Becton, Dickinson and Company, San Jose, CA, USA), according to the manufacturer's instructions. After treatment, $5 \times 10^{5}$ cells were collected by centrifugation at $1500 \mathrm{rpm}$ for $5 \mathrm{~min}$. The cell were re-suspended in $200 \mu \mathrm{L}$ of binding buffer and incubated with $5 \mu \mathrm{L}$ FITC Annexin V and $1 \mu \mathrm{L}$ of propidium iodide solution for $30 \mathrm{~min}$ at room temperature. Apoptosis was detected using a Calibur Flow Cytometer (Becton, Dickinson and Company, San Jose, CA, USA). Apoptotic cells were defined as annexin V-positive/propidium-negative.

\section{Hoechst 33258 staining assay}

After treatment, apoptotic cells were detected using Hoechst 33258 staining (Beyotime, Haimen, China), according to the manufacturer's protocols. Stained cells were observed under a fluorescent microscope using 350-nm excitation and 460-nm emission.

\section{Luciferase reporter assay}

The 3'-untranslated region (3'UTR) of E2F3 mRNA containing the predicted miR-449a binding site was amplified by PCR using the Takara PCR Amplification Kit (Takara, Dalian, China). The corresponding mutant constructs were created using the Quick Mutagenesis Stratagene kit (Stratagene, La Jolla, CA). PCR products were digested and cloned into the psiCHECK-2 reporter vector (Promega, USA). Cells (0.5 $\times 105)$ were seeded in 24-well plates and cultured for $24 \mathrm{~h}$. Reporter plasmids (200 ng psiCHECK-2-E2F3-wild or psiCHECK-2-E2F3-mut) and $100 \mathrm{nmol} / \mathrm{L} \mathrm{miR-449a} \mathrm{mimics} \mathrm{were} \mathrm{cotransfected} \mathrm{into} \mathrm{SGC7901} \mathrm{cells} \mathrm{using}$ Lipofectamine 2000 (Invitrogen, Grand Island, NY, USA). After $48 \mathrm{~h}$, the cells were lysed and reporter activity was determined using a Dual Luciferase Reporter Assay Kit (Promega, Madison, WI, USA), according to the manufacturer's instructions.

\section{Western blotting}

Western blotting was performed according to standard methods, as described previously [12]. Membranes were probed with polyclonal rabbit antibodies against anti-E2F3 (1:500; Abcam, Cambridge, MA, 
USA). The membranes were stripped and re-probed with an anti-GAPDH rabbit polyclonal antibody (Abcam, Cambridge, MA, USA) as a loading control. The blot was developed using enhanced chemiluminescence solution (Beyotime, Haimen, China) and photographed using the FluorChem imaging system (Alpha Innotech Corp., San Leandro, CA, USA). The intensity of each spot was analyzed with AlphaEaseFC software.

Statistical analysis

All data are represented as the mean \pm standard deviation of at least three independent experiments. The results were analyzed using a two-tailed Student's $t$ test. Results were considered statistically significant at $p<0.05$.

\section{Results}

MiR-449a expression is down-regulated in gastric cancer

To evaluate the role of miR-449a in the development of gastric cancer, we detected the expression levels of miR-449a in the human gastric cancer cell line SGC-7901 and normal human gastric epithelial cells (NGEC) using quantitative RT-PCR. As shown in Fig. 1A, miR449a expression was markedly down-regulated in SGC7901 cells compared with normal human gastric epithelial cells, as determined by real-time PCR analysis. To analyze the clinical relevance of this result, we extended our analysis to assess the expression of miR449a in a series of 12 human primary gastric cancer tissues and paired normal adjacent gastric tissues. Similar to gastric cancer cell lines, all tumor samples had lower levels of miR449a expression compared with paired normal tissues (Fig. 1B). These data confirm that $\mathrm{miR}-449 \mathrm{a}$ is down-regulated in gastric cancer and indicate that miR-449a may be a potential tumor suppressor.

MiR-449a suppresses growth and promotes apoptosis in SGC-7901 cells

To investigate the biological effect of miR-449a on gastric cancer, we evaluated the effect of miR-449a on the growth of SGC-7901 cells. As shown in Fig. 2A, CCK-8 assays revealed that ectopic expression of miR-449a significantly suppressed gastric cancer cell growth. Similar results were observed using colony formation assays (Fig. 2B and 2C). To explore the mechanism by which miR-449a inhibits cell proliferation, we analyzed the cell-cycle distribution in transfected cells using flow cytometry. miR-449a mimics induced significant G0/G1 arrest in miR-449a-overexpressing SGC-7901 cells compared with control cells (Fig. 2D and 2E). Furthermore, Annexin V and Hoechst 33258 staining (Fig. 2F, 2G and

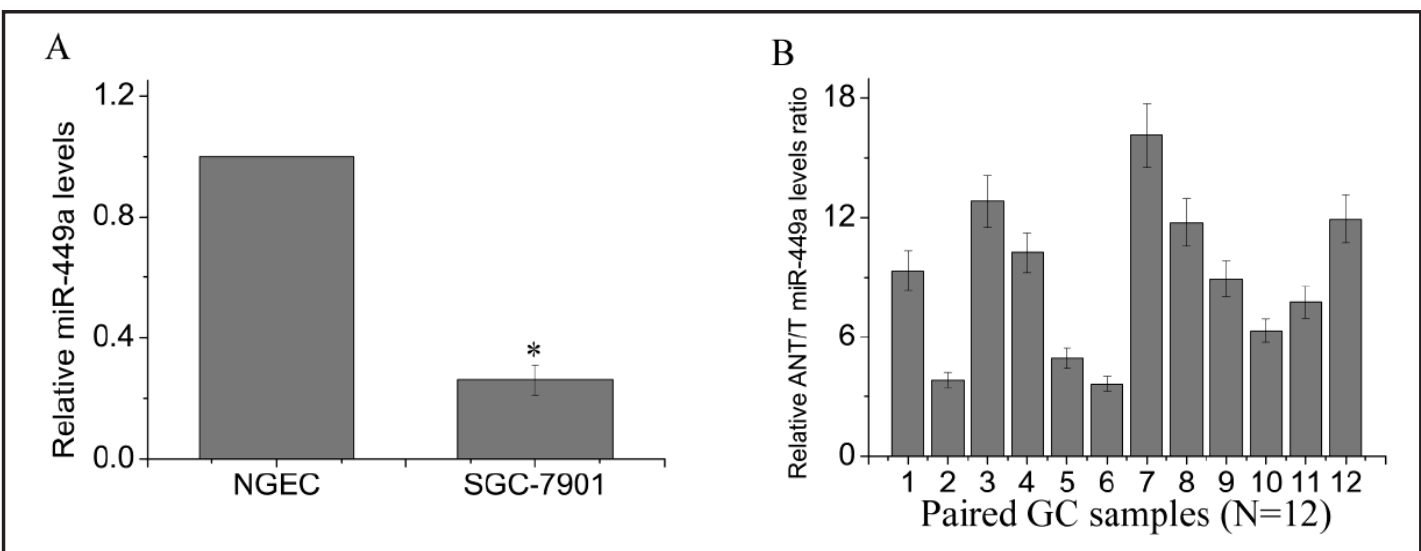

Fig. 1. MiR-449a is down-regulated in human gastric cancer cell lines and tissues. A: Real-time PCR analysis of miR-449a expression in NGEC and gastric cancer SGC-7901 cell lines. B: MiR-449a expression in primary gastric cancer tissues (T) compared to paired adjacent normal tissues (ANT) from 12 patients. Experiments were repeated at least three times. Bars denote the mean of three independent experiments. ${ }^{*} P<0.05$. 
A
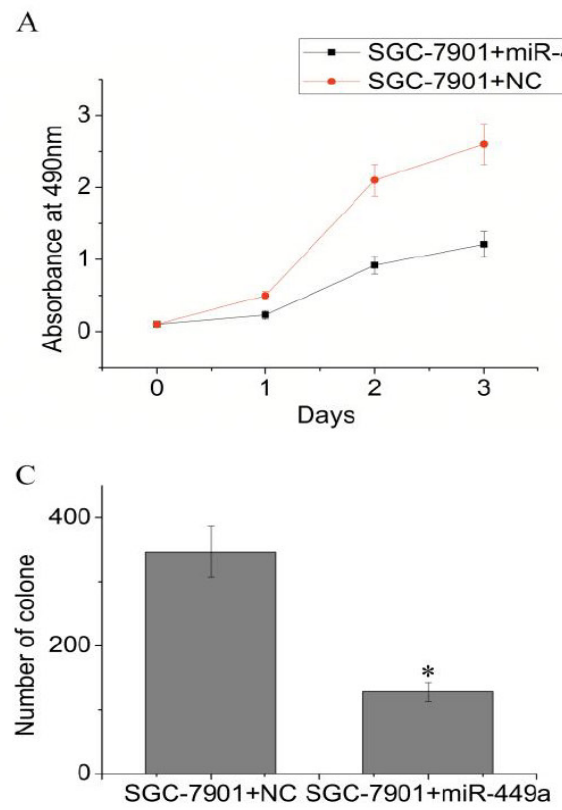

$\mathrm{E}$

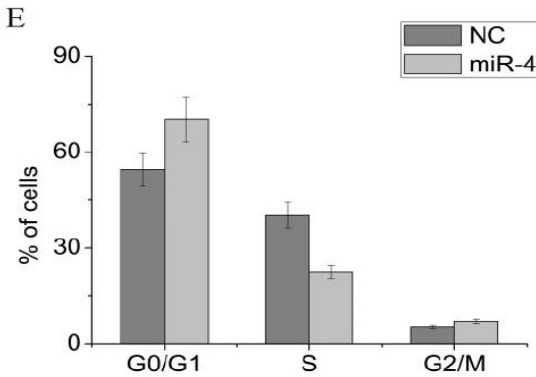

G

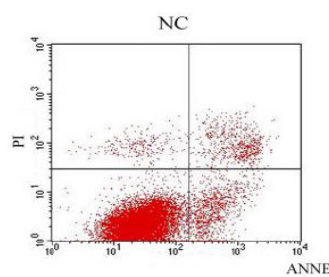

B
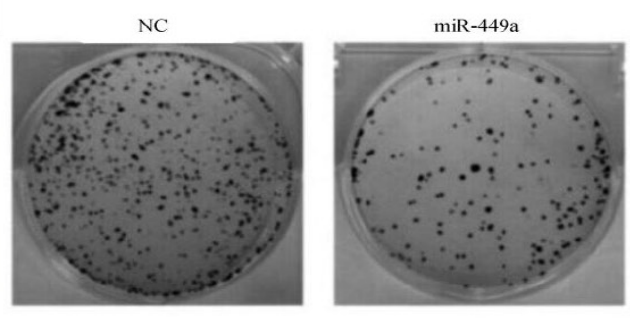

$\mathrm{D}$
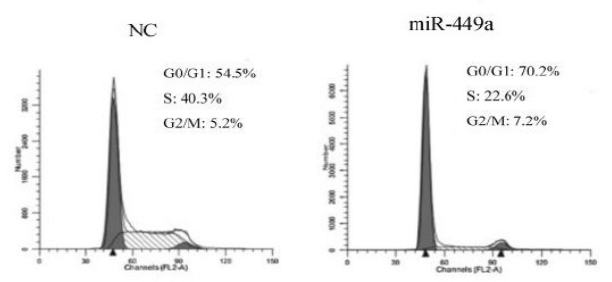

F
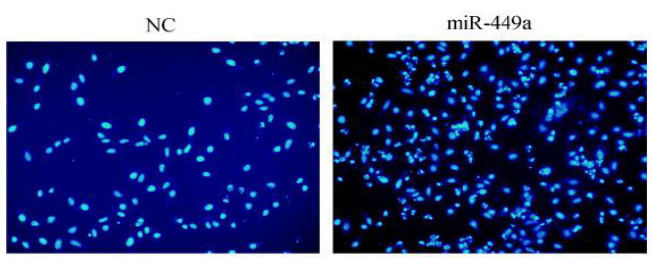

$\mathrm{H}$

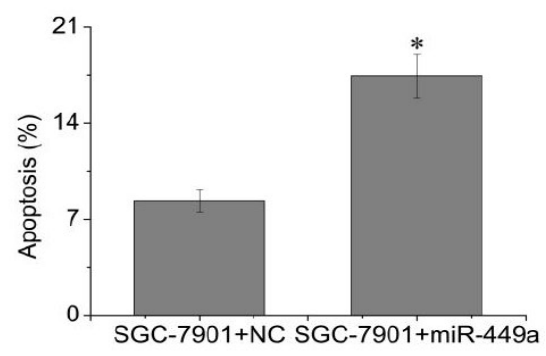

Fig. 2. MiR-449a suppresses growth and promotes apoptosis in SGC-7901 cells. A: Cell proliferation was measured using CCK-8 assays. Overexpression of miR-449a inhibited SGC-7901 cell proliferation. B, C: Colony formation ability was determined using colony formation assays. Overexpression of miR-449a inhibited the colony formation ability of SGC-7901 cells. D, E: Cell-cycle distribution was detected using flow cytometry. F: Apoptosis was detected using Hoechst 33258 staining assays. G, H: Apoptosis was detected by annexin-V FITC/PI double-staining. Overexpression of miR-449a enhanced apoptosis induced by $5 \mu \mathrm{M}$ cisplatin in SGC-7901 cells. ${ }^{*} P<0.05$.

$2 \mathrm{H}$ ) demonstrated that miR-449a overexpression promoted apoptosis in response to $5 \mu \mathrm{M}$ cisplatin. These results suggest that miR-449a is a tumor suppressor in gastric cancer cells in vitro.

E2F3 is a direct target of miR-449a in gastric cancer cells

Because E2F3 is associated with the development and progression of cancer, it is essential to elucidate regulatory mechanisms that converge on this molecule. Analysis using 
A

Position 2739-2737 of E2F3 3' UTR

hsa-miR-449a

5. ....canduanuUguahacacugcca...

3. $\quad$ UIIIIIII

$\mathrm{C}$

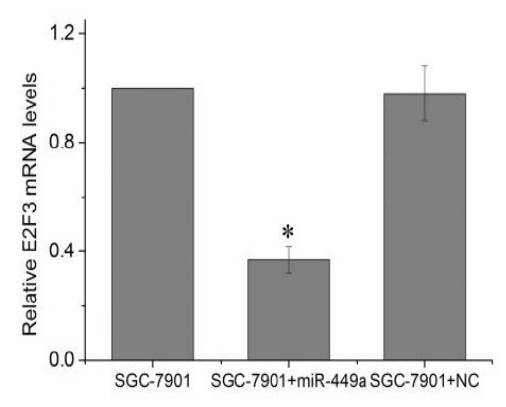

$\mathrm{E}$

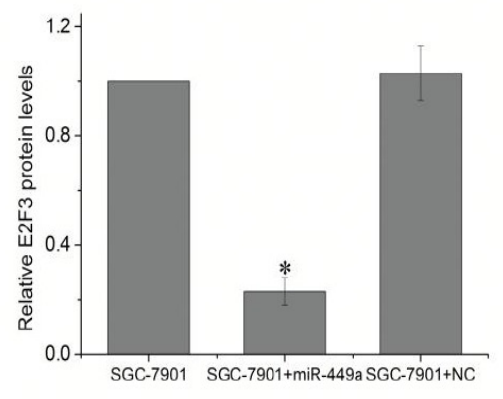

B

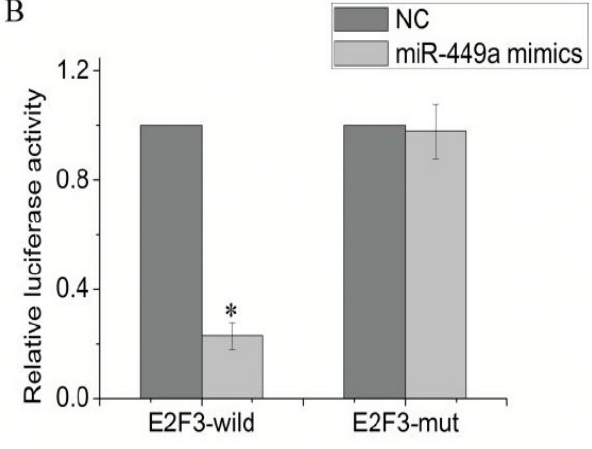

$\mathrm{D}$

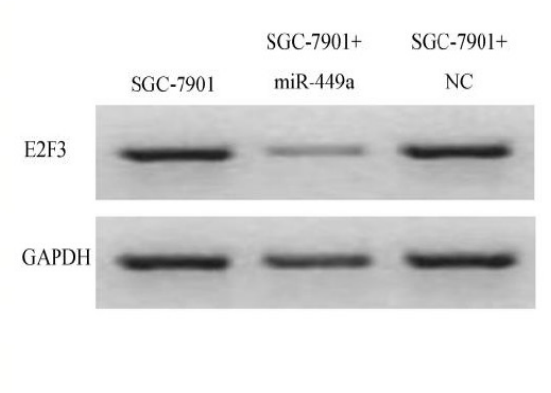

F
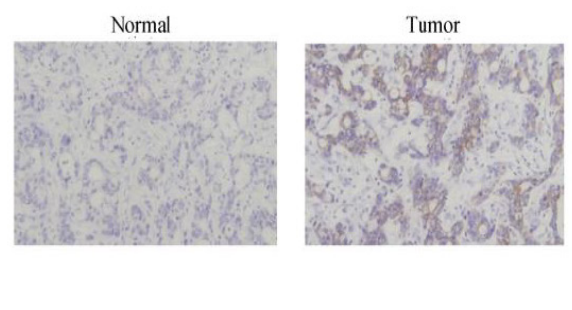

Fig. 3. E2F3 is a direct target of miR-449a in gastric cancer cells. A: TargetScan predicted a miR-449a target sequence in the 3'-UTR of E2F3 mRNA. B: E2F3-3'UTR-wild or E2F3-3'UTR-mut plasmids were transfected into miR-449a-treated SGC-7901 cells and assayed for luciferase activity. C: The mRNA levels of E2F3 were detected by RT-PCR in SGC-7901 cells transfected with miR-449a mimics or NC. D, E: E2F3 protein levels were detected by western blotting in SGC-7901 cells transfected with miR-449a mimics or NC. F: E2F3 expression was detected by immunohistochemistry in gastric cancer tissue and non-tumor gastric tissues. $* P<0.05$.

TargetScan Release 6.2 (http://www.targetscan.org/) indicated that E2F3 is a potential target of miR-449a (Fig. 3A), a miRNA that was previously reported to be down-regulated in various tumors. To validate E2F3 as a miR-124 target, we conducted luciferase reporter assays using E2F3-3'UTR-wild or E2F3-3'UTR-mut in miR-449a transfected SGC7901 cells (Fig. 3B). The luciferase activity of the E2F3-3'UTR-wild plasmid decreased (52\%) following treatment with miR-449a mimics, whereas the inhibitory effect of the miR-449a mimics was not observed with the mutated construct (Fig. 3B). These results indicate that miR-449a suppresses gene expression through binding the 3' UTR of E2F3 mRNA. Moreover, qRT-PCR and western blot analyses demonstrated that the expression of E2F3 mRNA and protein were decreased after treatment with miR-449a mimics in SGC-7901 cells (Fig. 3C, 3D and 3E). 


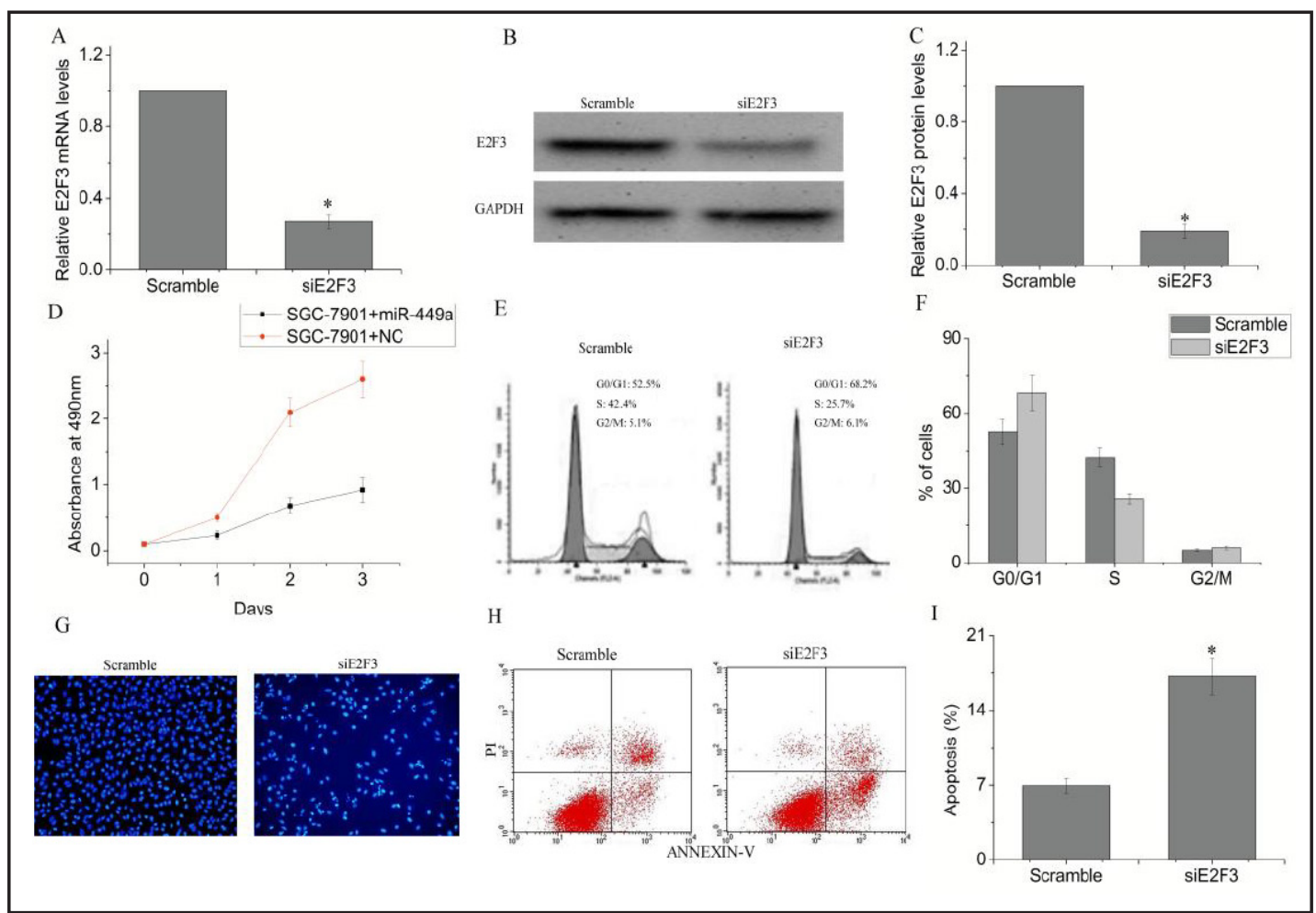

Fig. 4. MiR-449a mimics and siE2F3 elicit similar repressive effects. A: E2F3 mRNA levels were detected by RT-PCR in SGC-7901 cells transfected with siE2F3 or scrambled siRNA. B, C: E2F3 protein levels were detected by western blotting in SGC-7901 cells transfected with siE2F3 or scrambled siRNA. D: Cell proliferation was measured using CCK-8 assays in SGC-7901 cells transfected with siE2F3 or scrambled siRNA. E, F: Cell-cycle distribution was detected by flow cytometry in SGC-7901 cells transfected with siE2F3 or scrambled siRNA. G: Apoptosis was detected using Hoechst 33258 staining assays in SGC-7901 cells transfected with siE2F3 or scrambled siRNA. G, H: Apoptosis was detected using annexin-V FITC/PI double-staining in SGC-7901 cells transfected with siE2F3 or scrambled siRNA. ${ }^{*} P<0.05$.

In human tissue samples, E2F3 expression was up-regulated compared with paired normal tissues (Fig. 3F). Taken together, these results demonstrate that E2F3 is a direct target of miR-449a.

\section{MiR-449a mimics and siE2F3 elicit similar repressive effects}

To determine whether E2F3 plays an important role in gastric cancer cells, we transfected E2F3-specific siRNA (si-E2F3) into cells to knockdown E2F3 expression. As shown in Fig. $4 \mathrm{~A}, 4 \mathrm{~B}$ and $4 \mathrm{C}$, transfection with siE2F3 effectively reduced E2F3 at the mRNA and protein levels, as determined by RT-PCR and western blotting, respectively. Next, we treated the gastric cancer cells with scrambled or siE2F3 and evaluated cell viability. Treatment with the siE2F3 decreased SGC-7901 cells growth relative to scrambled siRNA (Fig. 4D). siE2F3transfected cells also displayed a similar growth arrest phenotype to that observed with miR-449a mimic treatment (Fig. 4E and 4F). Treatment with the siE2F3 promoted SGC-7901 cell apoptosis relative to scrambled siRNA (Fig. 4G, 4H and 4I). These data indicate that miR449a mimics and siE2F3 elicit similar repressive effects on SGC-7901 cells.

Overexpression of E2F3 reverses the repressive effects of miR-449a mimics

Next, we examined whether overexpression of E2F3 was able to reverse the repressive effects of miR-449a mimics. SGC-7901 cells were co-transfected with miR-449a mimics and pCDNA3.1-E2F3, which encodes the entire E2F3 coding sequence but lacks its normal 3 -untranslated sequence. CCK-8 assays revealed that E2F3 overexpression rescued the 


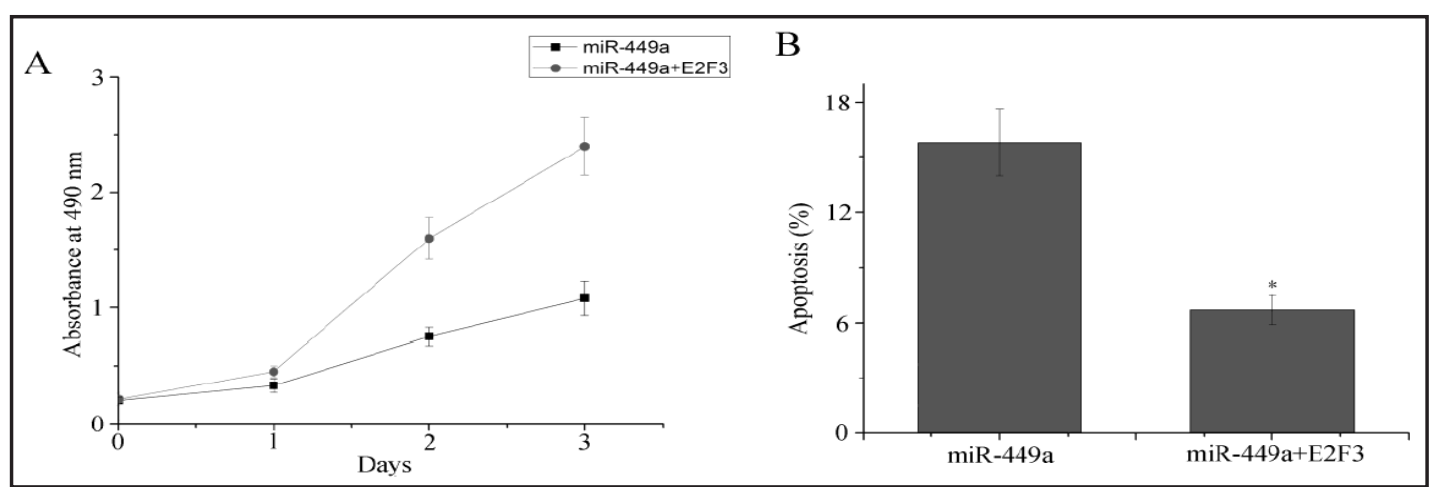

Fig. 5. Overexpression of E2F3 reverses the repressive effects caused by miR-449a mimics. A: Cell proliferation was measured using CCK-8 assays in SGC-7901 cells transfected with miR-449a mimics alone or co-transfected with miR-449a mimics and pCDNA3.1-E2F3. B: Apoptosis was detected by annexin-V FITC/PI double-staining in SGC-7901 cells transfected with miR-449a mimics alone or co-transfected with miR-449a mimics and pCDNA3.1-E2F3. ${ }^{*} P<0.05$.

growth-repressing effects of miR-449a mimics (Fig. 5A). Next, we examined the effect of E2F3 overexpression on SGC-7901 cells apoptosis. As shown in Fig. 5B, fewer apoptotic cells were detected after cotransfection with pCDNA3.1-E2F3 and miR-449a mimics compared to miR-449a alone. These data suggest that the repressive effects of miR-449a are in part facilitated by the down-regulation of E2F3.

\section{Discussion}

An abundance of in vivo and in vitro studies have demonstrated that microRNAs (miRNAs) regulate cancer development and progression in carcinogenesis as either tumor suppressors or oncogenes [13]. Despite increasing evidence pointing to a role for miR-449a as a tumor suppressor, the role of miR-449a in human gastric cancer remains unclear [1416]. In the present study, miR-449a expression was downregulated in gastric cancer tissues and cell lines. We also found that elevated miR-449a levels inhibited gastric cancer cell growth, which was associated with the induction of apoptosis and cell cycle arrest. Luciferase assays confirmed that miR-449a directly binds the 3'-UTR of E2F3 mRNA and inhibits E2F3 translation in gastric cancer cells.

The human miR-449 cluster is located on chromosome 5 in a highly conserved region within the second intron of the CDC20B gene, a homolog of CDC20 [17]. This cluster, which encodes the highly conserved miR-449a and miR-449b, contains similar sequences and secondary structures as the miR-34 family, so they were classified as one family of microRNAs [17]. Under normal conditions, high levels of miR-449a are present in lung, testis and trachea tissues [18]. Growing evidence indicates that deregulation of miRNAs plays an important role in human carcinogenesis $[19,20]$. miR-449a is downregulated and displays tumor suppressive effects in several malignant tumors, including lung [21], liver [22] and gastric [23] cancer; breast [24] and bladder carcinoma [7], prostatic carcinoma [25, 26], and oophoroma [27]. Kheir et al. confirmed that miR-449a expression is low or absent in 8 out of 10 primary gastric cancers [23]. Hu et al. demonstrated that miR-449a could modulate the cell cycle and apoptosis by regulating cyclin D1 and Bcl-2 expression in SGC7901 cells [16]. In the present study, we found that miR-449a is downregulated in gastric cancer cell lines and tumor tissues. Furthermore, miR-449a suppressed growth and promoted apoptosis in gastric cancer cells. These data indicate that miR-449a plays a tumor suppressor role in gastric cancer cells.

E2F-family transcription factors play important roles in cell cycle progression and cancer [28]. They are 8 members of the E2F-family: E2F1-E2F3 act as transcriptional activators and 


\section{Cellular Physiology Cell Physiol Biochem 2015;35:2033-2042 \begin{tabular}{ll|l} 
and Biochemistry & $\begin{array}{l}\text { DOI: 10.1159/000374010 } \\
\text { Published onlIne: VIarch 30, } 2015\end{array}$ & $\begin{array}{l}\text { C 2015 S. Karger AG, Basel } \\
\text { www.karger.com/cpb }\end{array}$ \\
\hline
\end{tabular}}

Li et al.: MicroRNA-449a Inhibits Proliferation and Induces Apoptosis in Gastric Cancer

E2F4-E2F8 are considered transcriptional repressors [28]. E2F activators regulate genes necessary for $\mathrm{G}_{1}$ - to S-phase transition and DNA replication. E2F3 acts as a transcriptional activator and increases cellular proliferation through the G1/S transition [29, 30]. Recent studies indicate that E2F3 modulates the expression of several genes, including Cdk1, Aurora-A, and Survivin [28, 31, 32]. In this study, E2F3 was confirmed to be a direct target gene of miR-449a using luciferase activity assays. Our data suggest that the down-regulation of E2F3 by miR-449a causes cell cycle arrest and apoptosis. In contrast, the suppressive effect of miR-449a on cell growth is largely due to the down-regulation of E2F3 expression, because the E2F3 silencing showed a smaller inhibitory effect on cell growth than ectopic miR-449a expression. Thus, down-regulation of E2F3 represents a molecular mechanism by which miR-449a exerts its tumor suppressor function.

In conclusion, our study revealed that miR-449a is frequently down-regulated in GC. miR449a inhibits GC cell growth and induces GC cell apoptosis. The tumor suppressor functions of miR-449a in GC involve the inhibition of cell proliferation, induction of G0/G1 arrest and apoptosis by directly targeting E2F3. There are some limitations to our research study. For example, there are no clinical data and no comparison to adjacent normal tissue in this study. Further studies should be carried out to concern the effects of miR-449a in gastric cancer patients with different stages of cancer. Additionally, the lack of use of other commonly used chemotherapeutic drug such as $5-\mathrm{FU}$, is also limitation of this study.

\section{Disclosure Statement}

None.

\section{References}

1 Herszenyi L, Tulassay Z: Epidemiology of gastrointestinal and liver tumors. Eur Rev Med Pharmacol Sci 2010;14:249-258.

-2 Kim SJ, Wang YG, Lee HW, Kang HG, La SH, Choi IJ, Irimura T, Ro JY, Bresalier RS, Chun KH: Up-regulation of neogenin-1 increases cell proliferation and motility in gastric cancer. Oncotarget 2014;5:3386-3398.

3 Ren J, Huang HJ, Gong Y, Yue S, Tang LM, Cheng SY: MicroRNA-206 suppresses gastric cancer cell growth and metastasis. Cell Biosci 2014;4:26.

4 Li Q Wang JX, He YQ, Feng C, Zhang XJ, Sheng JQ, Li PF: MicroRNA-185 regulates chemotherapeutic sensitivity in gastric cancer by targeting apoptosis repressor with caspase recruitment domain. Cell Death Dis 2014;5:e1197.

5 Calin GA, Croce CM: MicroRNA signatures in human cancers. Nat Rev Cancer 2006;6:857-866.

6 Ishiguro H, Kimura M, Takeyama H: Role of microRNAs in gastric cancer. World J Gastroenterol 2014;20:5694-5699.

7 Chen H, Lin YW, Mao YQ, Wu J, Liu YF, Zheng XY, Xie LP: MicroRNA-449a acts as a tumor suppressor in human bladder cancer through the regulation of pocket proteins. Cancer Lett 2012;320:40-47.

-8 Smoot DT, Sewchand J, Young K, Desbordes BC, Allen CR, Naab T: A method for establishing primary cultures of human gastric epithelial cells. Methods Cell Sci 2000;22:133-136.

9 Chen P, Huang Y, Zhang B, Wang Q, Bai P: EphA2 enhances the proliferation and invasion ability of LNCaP prostate cancer cells. Oncol Lett 2014;8:41-46.

10 Madhavan J, Mitra M, Mallikarjuna K, Pranav O, Srinivasan R, Nagpal A, Venkatesan P, Kumaramanickavel G: KIF14 and E2F3 mRNA expression in human retinoblastoma and its phenotype association. Mol Vis 2009;15:235-240.

11 Liu T, Hou L, Huang Y: EZH2-specific microRNA-98 inhibits human ovarian cancer stem cell proliferation via regulating the pRb-E2F pathway. Tumour Biol 2014;35:7239-7247.

12 Iyer G, Milowsky MI: Fibroblast growth factor receptor-3 in urothelial tumorigenesis. Urol Oncol 2013;31:303-311. 


\section{Cellular Physiology Cell Physiol Biochem 2015;35:2033-2042

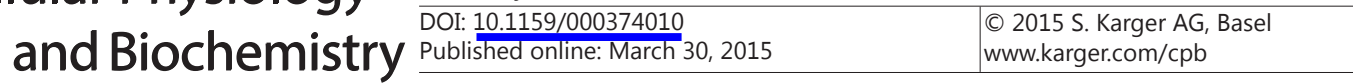

13 Tian L, Li M, Ge J, Guo Y, Sun Y, Liu M, Xiao H: MiR-203 is downregulated in laryngeal squamous cell carcinoma and can suppress proliferation and induce apoptosis of tumours. Tumour Biol 2014;35:59535963.

14 Ye W, Xue J, Zhang Q, Li F, Zhang W, Chen H, Huang Y, Zheng F: MiR-449a functions as a tumor suppressor in endometrial cancer by targeting CDC25A. Oncol Rep 2014;32:1193-1199.

15 Ren XS, Yin MH, Zhang X, Wang Z, Feng SP, Wang GX, Luo YJ, Liang PZ, Yang XQ, He JX, Zhang BL: Tumorsuppressive microRNA-449a induces growth arrest and senescence by targeting E2F3 in human lung cancer cells. Cancer Lett 2014;344:195-203.

16 Hu J, Fang Y, Cao Y, Qin R, Chen Q: miR-449a regulates proliferation and chemosensitivity to cisplatin by targeting cyclin D1 and BCL2 in SGC7901 cells. Dig Dis Sci 2014;59:336-345.

17 Lize M, Klimke A, Dobbelstein M: MicroRNA-449 in cell fate determination. Cell Cycle 2011;10:2874-2882.

18 Lize M, Pilarski S, Dobbelstein M: E2F1-inducible microRNA 449a/b suppresses cell proliferation and promotes apoptosis. Cell Death Differ 2010;17:452-458.

19 Lu J, Getz G, Miska EA, Alvarez-Saavedra E, Lamb J, Peck D, Sweet-Cordero A, Ebert BL, Mak RH, Ferrando AA, Downing JR, Jacks T, Horvitz HR, Golub TR: MicroRNA expression profiles classify human cancers. Nature 2005;435:834-838.

20 Volinia S, Calin GA, Liu CG, Ambs S, Cimmino A, Petrocca F, Visone R, Iorio M, Roldo C, Ferracin M, Prueitt RL, Yanaihara N, Lanza G, Scarpa A, Vecchione A, Negrini M, Harris CC, Croce CM: A microRNA expression signature of human solid tumors defines cancer gene targets. Proc Natl Acad Sci U S A 2006;103:22572261.

-21 Jeon HS, Lee SY, Lee EJ, Yun SC, Cha EJ, Choi E, Na MJ, Park JY, Kang J, Son JW: Combining microRNA-449a/b with a HDAC inhibitor has a synergistic effect on growth arrest in lung cancer. Lung Cancer 2012;76:171176.

-22 Buurman R, Gurlevik E, Schaffer V, Eilers M, Sandbothe M, Kreipe H, Wilkens L, Schlegelberger B, Kuhnel F, Skawran B: Histone deacetylases activate hepatocyte growth factor signaling by repressing microRNA-449 in hepatocellular carcinoma cells. Gastroenterology 2012;143:811-820 e811-815.

-23 Bou Kheir T, Futoma-Kazmierczak E, Jacobsen A, Krogh A, Bardram L, Hother C, Gronbaek K, Federspiel B, Lund AH, Friis-Hansen L: miR-449 inhibits cell proliferation and is down-regulated in gastric cancer. Mol Cancer 2011;10:29.

24 Yang X, Feng M, Jiang X, Wu Z, Li Z, Aau M, Yu Q: miR-449a and miR-449b are direct transcriptional targets of E2F1 and negatively regulate pRb-E2F1 activity through a feedback loop by targeting CDK6 and CDC25A. Genes Dev 2009;23:2388-2393.

25 Noonan EJ, Place RF, Pookot D, Basak S, Whitson JM, Hirata H, Giardina C, Dahiya R: miR-449a targets HDAC-1 and induces growth arrest in prostate cancer. Oncogene 2009;28:1714-1724.

-26 Noonan EJ, Place RF, Basak S, Pookot D, Li LC: miR-449a causes Rb-dependent cell cycle arrest and senescence in prostate cancer cells. Oncotarget 2010;1:349-358.

27 Zhang Q, He XJ, Ma LP, Li N, Yang J, Cheng YX, Cui H: [Expression and significance of microRNAs in the p53 pathway in ovarian cancer cells and serous ovarian cancer tissues]. Zhonghua Zhong Liu Za Zhi 2011;33:885-890.

28 Chen HZ, Tsai SY, Leone G: Emerging roles of E2Fs in cancer: an exit from cell cycle control. Nat Rev Cancer 2009;9:785-797.

29 Wu L, Timmers C, Maiti B, Saavedra HI, Sang L, Chong GT, Nuckolls F, Giangrande P, Wright FA, Field SJ, Greenberg ME, Orkin S, Nevins JR, Robinson ML, Leone G: The E2F1-3 transcription factors are essential for cellular proliferation. Nature 2001;414:457-462.

-30 Sharma N, Timmers C, Trikha P, Saavedra HI, Obery A, Leone G: Control of the p53-p21CIP1 Axis by E2f1, E2f2, and E2f3 is essential for G1/S progression and cellular transformation. J Biol Chem 2006;281:3612436131.

-31 He L, Yang H, Ma Y, Pledger WJ, Cress WD, Cheng JQ: Identification of Aurora-A as a direct target of E2F3 during G2/M cell cycle progression. J Biol Chem 2008;283:31012-31020.

-32 Jiang Y, Saavedra HI, Holloway MP, Leone G, Altura RA: Aberrant regulation of survivin by the RB/E2F family of proteins. J Biol Chem 2004;279:40511-40520. 